\title{
Long noncoding RNA MALAT1 regulates autophagy associated chemoresistance via miR-23b-3p sequestration in gastric cancer
}

\author{
Hu YiRen ${ }^{1}$, Yu YingCong ${ }^{2,3}$, You Sunwu', Li Keqin ${ }^{1}$, Tong Xiaochun ${ }^{1}$, Chen Senrui ${ }^{1}$, Chen Ende ${ }^{1}$, Lin XiZhou ${ }^{2,3}$ \\ and Chen Yanfan ${ }^{4^{*}}$
}

\begin{abstract}
Background: Chemoresistance has long been recognized as a major obstacle in cancer therapy. Clarifying the underlying mechanism of chemoresistance would result in novel strategies to improve patient's response to chemotherapeutics.

Methods: IncRNA expression levels in gastric cancer (GC) cells was detected by quantitative real-time PCR (qPCR). MALAT1 shRNAs and overexpression vector were transfected into GC cells to down-regulate or up-regulate MALAT1 expression. In vitro and in vivo assays were performed to investigate the functional role of MALAT1 in autophagy associated chemoresistance.

Results: We showed that chemoresistant GC cells had higher levels of MALAT1 and increased autophagy compared with parental cells. Silencing of MALAT1 inhibited chemo-induced autophagy, whereas MALAT1 promoted autophagy in gastric cancer cells. Knockdown of MALAT1 sensitized GC cells to chemotherapeutics. MALAT1 acts as a competing endogenous RNA for miR-23b-3p and attenuates the inhibitory effect of miR-23b-3p on ATG12, leading to chemo-induced autophagy and chemoresistance in GC cells.
\end{abstract}

Conclusions: Taken together, our study revealed a novel mechanism of IncRNA-regulated autophagy-related chemoresistance in GC, casting new lights on the understanding of chemoresistance.

Keywords: InCRNA, MALAT1, Gastric cancer, Chemoresistance, Autophagy

\section{Background}

Gastric cancer (GC) is the fifth most frequent cancer and the third common cause of cancer-related death worldwide $[1,2]$. Surgical resection remains the only curative treatment, but most of the patients that suffer from GC are diagnosed at advanced stages $[3,4]$. Chemotherapy is the first-line treatment for these patients. However, despite the advancements in the development of novel chemotherapeutic drugs, chemotherapy has only modest efficacy in patients with advanced/metastatic GC. Chemoresistance has long been recognized as a major obstacle in cancer therapy. Thus, the identification of

\footnotetext{
* Correspondence: chenyanfan8659@sina.com

${ }^{4}$ Department of radiology, Wenzhou No.3 Clinical Institute of Wenzhou Medical University, Wenzhou People's Hospital, No. 57 Canghou Street, Wenzhou, Zhejiang 325000, China

Full list of author information is available at the end of the article
}

novel molecular mechanisms underlying chemoresistance may improve clinical outcome.

Previous studies have revealed that both intrinsic and acquired chemoresistance come from the genetic and epigenetic modifications occurring in cancer cells [5]. Although the factors responsible for chemoresistance can be diversified, it is well recognized that a cell's capability to manage stress plays a vital role. Accumulating evidence has demonstrated that autophagy, a favored survival strategy that help cancer cells overcome stressful conditions, may play an important role in chemoresistance [6].

Long noncoding RNAs are a class of long $(>200$ nucleotides) noncoding RNA molecules and have been shown to be a crucial player in cancer biology, including chemoresistance [7-9]. Metastasis-associated lung adenocarcinoma transcript 1 (MALAT1) is located on chromosome $11 \mathrm{q} 13$ and has been identified to be involved in a wide 
range of biological and cellular processes, including glycolysis [10], carcinogenesis [11], retinal neurodegeneration [12] and vascular growth [13]. However, how MALAT1 functions to therapeutically impact $\mathrm{GC}$ and the underlying mechanism remains largely unknown.

In the present study, we showed that MALAT1 is maintained at a higher expression level in chemoresistant GC cells than in chemosensitive cells. Additionally, our data indicated that knockdown of MALAT1 can sensitize GC cells to chemotherapy by blocking chemotherapy-induced autophagy. Mechanistically, MALAT1 competitively sequesters miR-23b-3p and relieves the inhibitory effect of miR-23b-3p on ATG12, thereby increasing the expression of ATG12. Thus, our study identified a novel role of MALAT1 in the regulation of autophagy and suggests that the knockdown of MALAT1 may sensitize GC cells to chemotherapeutics via suppression of chemotherapy-induced autophagy.

\section{Methods}

\section{Cell culture}

Two human gastric adenocarcinoma cell lines SGC7901 and BGC823 were used in this study. SGC7901/VCR cells were cultured as described previously [14, 15]. SGC7901 and BGC823 were purchased from Cell Bank of the Chinese Academy of Sciences (Shanghai, China). These cell lines were immediately expanded and frozen so that a new aliquot could be thawed every 3 to 4 months from a frozen vial of the same batch of cells. Cells were cultured in Dulbecco's modified Eagle's medium (DMEM, Gibco, Carlsbad, CA, USA) supplemented with $10 \%$ fetal calf serum, penicillin, and streptomycin (HyClone, Logan, UT, USA) at $37{ }^{\circ} \mathrm{C}$ in an atmosphere containing $5 \% \mathrm{CO}_{2}$.

\section{RNA preparation and quantitative real-time PCR}

Briefly, total RNA was extracted from tissues or cells using TRIzol reagent (Invitrogen, Carlsbad, CA, USA). The quality of total RNA was detected at an A260/A280 ratio using 1\% agarose gel electrophoresis. The GoScript Reverse Transcription System (Promega, Madison, Wis) was used to generate complementary DNA. The cDNA template was amplified by real-time RT-PCR using the SYBR Premix Dimmer Eraser kit (TaKaRa, Dalian, China). Gene expression was normalized to GADPH or U6 expression. The real-time PCRs were performed in triplicate and calculated by the $2^{-\Delta \Delta C t}$ method. Primers used in this study are listed in Additional file 1: Table S1.

\section{Western blot analysis}

Briefly, the total cellular protein was isolated with RIPA cell lysis buffer supplemented with protease inhibitors. Cytosolic protein was isolated using the Mitochondrial and Cytoplasmic Extraction Kit (Thermo Fisher Scientific, Rockford, IL). Protein content was determined by the
Bradford assay. Equal amounts $(30-50 \mu \mathrm{g})$ of proteins were separated by $10 \%$ sodium dodecyl sulfate/polyacrylamide gel electrophoresis and transferred to a PVDF Immobilon$\mathrm{P}$ membrane (Millipore, MA). After blocking with 5\% skim milk, the membrane was then incubated with indicated primary antibodies and secondary antibodies conjugated to horseradish peroxidase. Antibody-bound proteins were detected by ECL (enhanced chemiluminescence) Western Blotting Substrate (Pierce, Rockford, IL). The band intensity of the western blots and the normalization was analyzed using the ImageJ program (National Institutes of Health, Bethesda, MD). The primary antibodies used include rabbit polyclonal anti-human LC3B (1:500, Abcam), p62 (1:500, Abcam), ATG12 (1:800, Abcam), rabbit monoclonal anti-human caspase-3 (1:500, Abcam), caspase-9 (1:500, Abcam), rabbit monoclonal anti-human cytochrome C (1:500, Epitomics), EZH2 (1:500, Epitomics) and rabbit polyclonal anti-human Actin (1:4,000, Abcam). HRP-conjugated goat anti-rabbit IgG antibody (Abcam) was used as the secondary antibody.

\section{Electron microscopy}

Cells were treated as indicated and fixed with $2.5 \%$ glutaraldehyde containing $0.1 \mathrm{~mol} / \mathrm{L}$ sodium cacodylate. Samples were fixed using $1 \%$ osmium tetroxide, followed by dehydration with an increasing concentration gradient of ethanol and propylene oxide. Samples were then embedded, cut into $50-\mathrm{nm}$ sections, and stained with 3\% uranyl acetate and lead citrate. Images were acquired using a CM-120 electron microscope (PHILIPS).

\section{In vitro and in vivo drug-sensitivity assay}

For the in vitro drug-sensitivity assay, GC cells were seeded into 96-well plates at a density of $1 \times 10^{5}$ cells per well. The culture medium containing different concentrations of 5-fluorouracil (5-FU), VCR or cisplatin (CDDP) was added to each well. Forty-eight hours post cultivation, CCK-8 solution $(10 \mu \mathrm{l}$ per $100 \mu \mathrm{l}$ of medium in each well) was added to each well and incubated for $2 \mathrm{~h}$. The absorbance was measured by scanning with a microplate reader (MRX; Dynex Technologies, West Sussex, United Kingdom) at $450 \mathrm{~nm}$. Each group comprised six replicates, and the experiments were repeated at least 3 times. Then, the IC50 values for each drug were calculated.

All animal experiments were performed in the animal laboratory center of Wenzhou No.3 Clinical Institute of Wenzhou Medical University, Wenzhou People's Hospital and in accordance with the Guide for the Care and Use of Laboratory Animals published by the US National Institutes of Health (NIH publication number 85-23, revised 1996) and ARRIVE.

For in vivo experiments, SGC7901/VCR cells $\left(1 \times 10^{7}\right)$ transfected with the desired vector were subcutaneously injected into the flank area of 4-week-old female athymic 
nude mice ( $n=4$ mice per group). After two weeks, the mice were intraperitoneally injected with CDDP in PBS $(10 \mathrm{mg} / \mathrm{kg})$ once every week. Tumor volumes were measured every week with the following formula: $0.5 \times$ length $\times$ width $^{2}$ every week. Four weeks post inoculation, the mice were sacrificed and the tumors were photographed.

\section{Quantification of MALAT1 and miR-23b-3p expression levels} The copy number of MALAT1 and miR-23b-3p transcripts per cell was quantified by using a quantitative real-time RT-PCR assay as we described previously [9].

\section{Plasmid construction, lentiviral construction, and cell transfections}

Detailed descriptions of plasmid construction, lentiviral vector construction, and cell transfections can be found in Additional file 2.

\section{Luciferase reporter assay}

The fragments of MALAT1 containing the putative hsa-miR-23b-3p binding site were chemically synthesized. The corresponding mutants were created by mutating the hsa-miR-23b-3p seed region binding site. Cotransfection of psicheck2, psicheck2-MALAT1 wt or psicheck2- MALAT1-mut (miR-23b-3p) with miR23b-3p mimics, inhibitors or miRNA NC into GC cells was completed with Lipofectamine-mediated gene transfer. Forty-eight hours after transfection, using $100 \mu \mathrm{l}$ of passive buffer, cells were collected to detect the Renilla luciferase activity with the Dual-Luciferase Reporter Assay System (Promega) in TD-20/20. Forty-eight hours post transfection, the relative luciferase activity was determined after normalizing to Renilla luciferase activity.

\section{Cytosolic/nuclear fractionation isolation and biotin pull-down assay}

Detailed descriptions of the cytosolic/nuclear fractionation isolation and Biotin pull-down assays can be found in Additional file 2.

\section{Patients and clinical samples}

Written consent was obtained from all patients in this study. The human tissue specimens used in this study were approved by and under the censorship of the local ethics committee at Wenzhou No.3 Clinical Institute of Wenzhou Medical University, Wenzhou People's Hospital. Six GC patients, who received neoadjuvant chemotherapy before surgery between 2014 and 2015, were identified from Wenzhou People's Hospital, including 3 chemosensitive and 3 chemoresistant cases. The human specimens were subject to immunohistochemical staining of ATG12 and in situ hybridization of MALAT1.

\section{Statistical analysis}

All statistical analyses were performed utilizing SPSS version 17.0 software (Chicago, IL, USA). All data are presented as the mean \pm standard deviation from three independent repeats. Unless otherwise noted, the differences between two groups were analyzed using Student's $t$-test.

\section{Results}

Chemoresistant GC cells demonstrate blunted chemosensitivity compared to parental cells

The established chemoresistant cell lines SGC7901/VCR (vincristine) derived from human GC cell line SGC7901 was obtained from Professor D. Fan $[14,15]$. To confirm the chemosensitivity of SGC7901/VCR cells compared to SGC7901 cells, CCK-8 assays were performed to measure changes in cell proliferation and viability. As demonstrated in Fig. 1a, SGC7901/VCR cells had an enhanced resistance to cisplatin compared to SGC7901 cells. It is worth noting that SGC7901 and SGC7901/VCR demonstrated similar proliferation rates in the absence of chemotherapeutics (Fig. 1b).

\section{Chemoresistant GC cells exhibit increased autophagy}

Autophagy, a conserved lysosome-mediated intracellular degradation system, could protect cancer cells during stress conditions [16]. Autophagy can be induced by chemotherapeutics, and this process relieves cancer cells from chemotherapy-associated cellular damage, thereby contributing to chemoresistance [17]. Thus, we hypothesized that chemoresistant GC cells may have increased autophagy. We utilized transmission electron microscopy (TEM) and western blot analysis to evaluate autophagosomes. TEM demonstrated a substantial increase in the accumulation of autophagic vesicles in SGC7901/VCR cells compared with parental cells (Fig. 2a). LC3 was used as a measure of autophagy activation and the conversion of LC3-I to LC3-II is regarded as a hallmark of autophagy. P62, an adaptor protein that interacts with LC3-II , is also considered as a hallmark of autophagy [18]. As expected, SGC7901/VCR cells expressed a higher LC3-II to LC3-I ratio and a decreased expression level of p62 compared with SGC7901 cells (Fig. 2b). SGC7901/VCR cells were treated with chloroquine (CQ), an autophagy-lysosomal inhibitor, and a further elevation in the LC3-II level was observed, indicating an increase in the autophagic flux of chemoresistant cells (Fig. 2c). Next, we sought to explore whether chemotherapy-induced autophagy affects chemotherapeutic efficacy. We treated SGC7901/VCR cells with CQ and found that co-treatment of SGC7901/VCR with CQ $(5 \mu \mathrm{M})$ and cisplatin caused greater cytotoxicity than treatment with cisplatin alone as evidenced by the decreased IC50 concentration (Fig. 2d) and increased apoptosis rates (Fig. 2e and Additional file 1: Figure S1). 

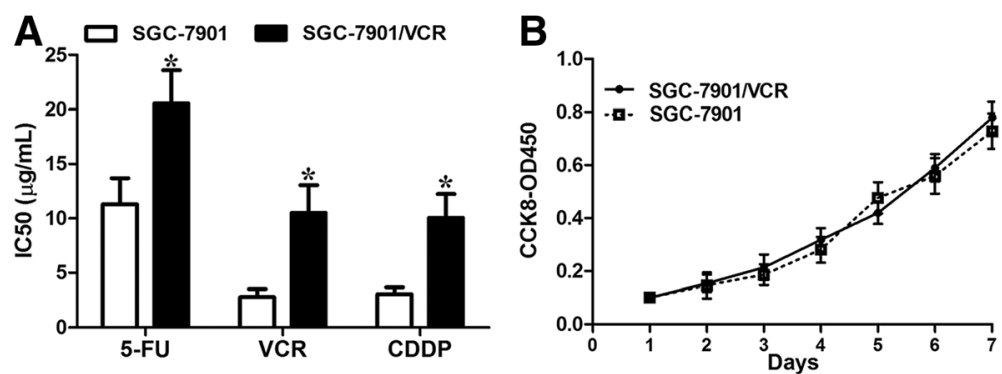

Fig. 1 SGC7901/NCR had increased chemoresistance. a SGC7901NCR cells harbored increased IC50 values compared with SGC7901 cells in response to chemotherapeutics. b The cell growth rates were determined by performing CCK-8 proliferation assays. SGC7901/VCR cells had similar cell proliferation rate, relative to control cells, in the absence of chemotherapeutics. The data are presented as the means \pm S.D. of values obtained in 3 independent experiments. ${ }^{*}, p<0.05$

\section{MALAT1 promotes autophagy-associated chemoresistance in GC cells}

We hypothesized that IncRNAs may play a role in the regulation of autophagy. We first determined the expression of several well-known lncRNAs (lincRNA-p21, HOTAIR, MALAT1, H19, linc-ROR, lncTCF7, lncRNA$A T B, B C 032469, L E T, G A P L I N C, N E A T 1$, and A7) in the SGC7901 and SGC7901/VCR cell lines using realtime quantitative real-time polymerase chain reaction (qRT-PCR) analysis. Of these lncRNAs, MALAT1 was expressed at higher levels in SGC7901/VCR cells than in SGC7901 cells (Fig. 3a). MALAT1 is upregulated in a wide range of types of cancer, including $\mathrm{GC}$, according to the Cancer Genome Atlas (TCGA) database (Additional file 1: Figure S2). Similar results were obtained from data in the Gene Expression Omnibus (GEO) database (GES50710, GSE47850, GSE58828), showing that the expression level of MALAT1 was higher in GC tissues than that in adjacent normal tissues. As MALAT1 was frequently upregulated in GC and can be induced by chemotherapy, we wanted to explore whether the upregulation of MALTA1 might play a role in the regulation of chemotherapeutic efficacy via promoting autophagy. We constructed MALAT1 stably knocked-down cells with an shRNA sequence targeting MALAT1 that has been shown to be efficient in a number of studies $[19,20]$. We found that knockdown of MALAT1 (Additional file 1: Figure S3a) greatly inhibited autophagy, as indicated by the attenuated LC3-II to LC3-I ratio, p62 protein level (Fig. 3b) and LC3 dots (Fig. 3c). In the functional aspect, we found that
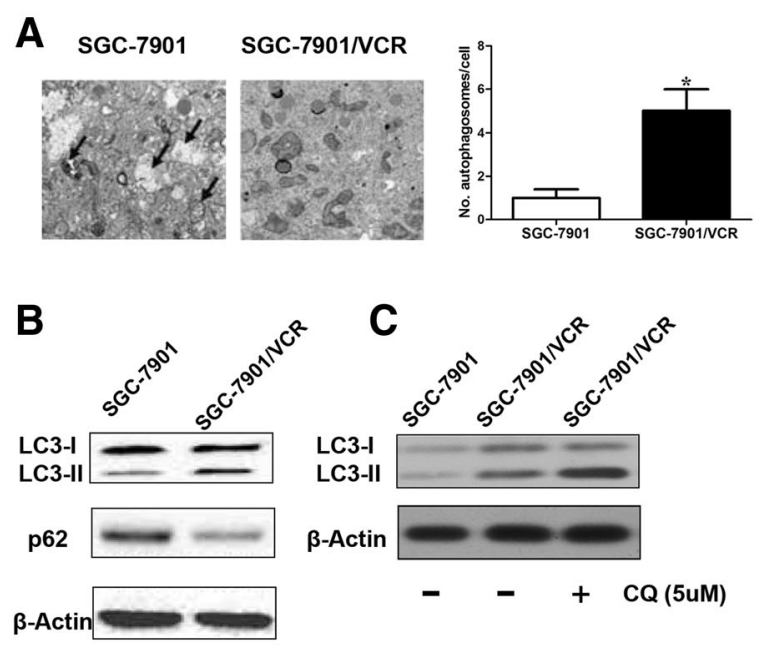
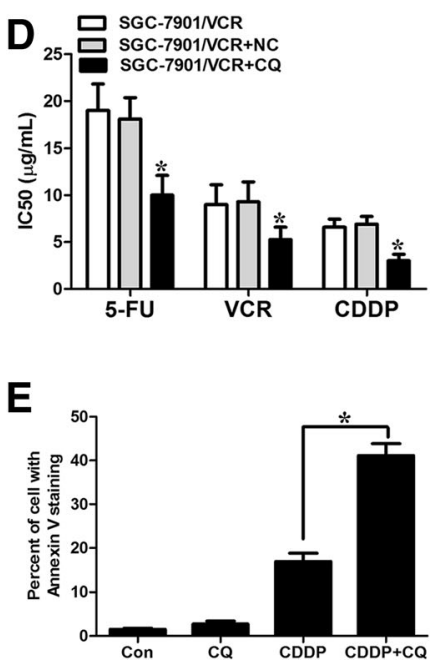

Fig. 2 SGC7901/NCR cells exhibit increased autophagy. a Autophagy was evaluated in SGC7901/NCR cells that exhibited chemoresistance using transmission electron microscopy. The data were quantified by counting the number of autophagosomes per cross-sectioned cell. $\mathbf{b}$ Autophagosome formation in whole cell lysates was determined by Western blot analysis using LC3 and p62 antibodies. The top band (16 kilodaltons) represents LC3-I, and the bottom band (14 kilodaltons) represents LC3-II. c SGC7901/NCR cells and their parental cell lines were treated with 10 mmol/L CQ for 24 hours before being subjected to Western blot analysis for LC3 expression. $\mathbf{d}$ CQ greatly enhanced the sensitivity of SGC7901/NCR cells to chemotherapeutic agents. e flow cytometric analysis of Annexin $\vee$ staining. The data are presented as the means \pm S.D. of values obtained in 3 independent experiments. $*, p<0.05$ 

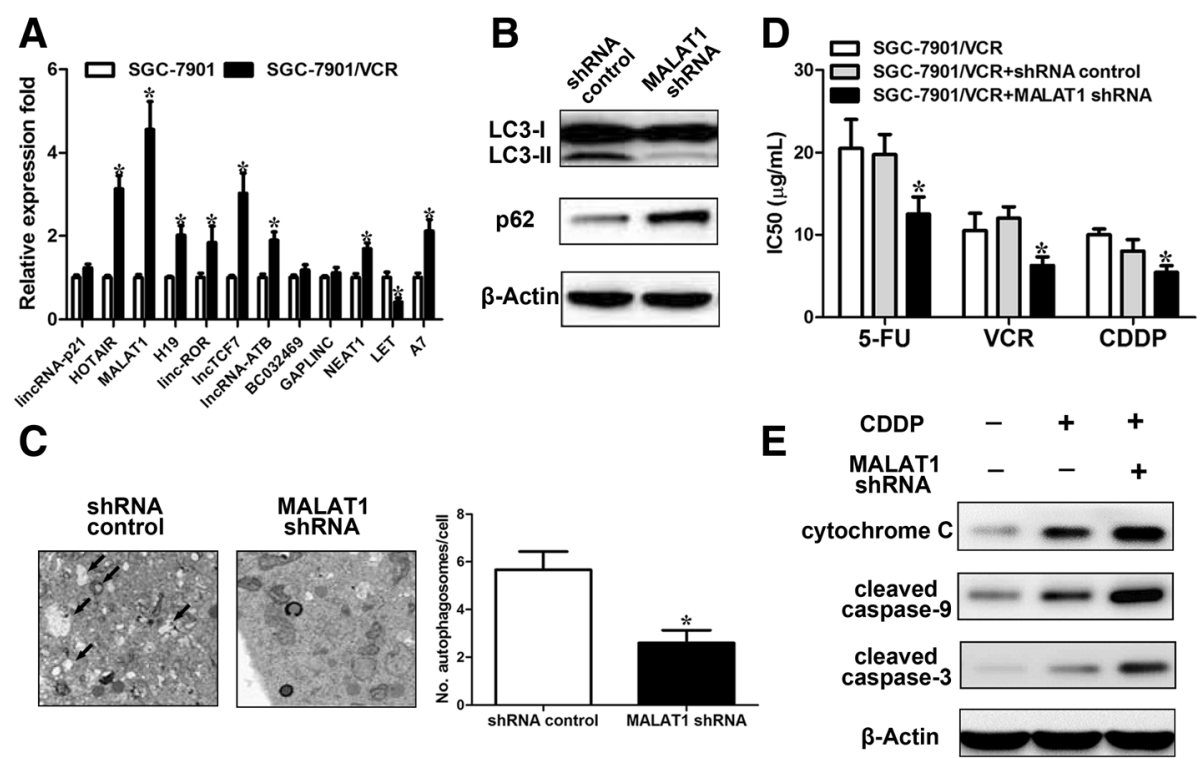

Fig. 3 MALAT1 promotes autophagy in GC cells. a Relative mRNA levels of specific IncRNAs in SGC7901NCR and SGC7901 cells using real-time PCR. b SGC7901NCR cells stably transfected with shRNA-MALAT1 or a control were subjected to Western blot analysis of LC3-II and p62. c Autophagy was evaluated using transmission electron microscopy in SGC7901/NCR stably transfected with shRNA-MALAT1 or a control. d Silencing of MALAT1 sensitized SGC7901NCR cells to chemotherapeutic agents as evidenced by decreased IC50 values. e SGC7901/VCR cells were treated with the cisplatin $(10 \mathrm{\mu g} / \mathrm{ml})$ for $24 \mathrm{~h}$. Total protein as well as cytoplasmic protein fractions were isolated, and the indicated proteins were detected by Western blot. The data are presented as the means \pm S.D. of values obtained in 3 independent experiments. ${ }^{*}, p<0.05$

knockdown of MALAT1 sensitized SGC7901/VCR cells to cisplatin as illustrated by decreased IC50 concentration (Fig. 3d) and increased expression levels of cleaved caspase3 and caspase-9 (Fig. 3e). Furthermore, overexpression of MALAT1 (Additional file 1: Figure S3b) enhanced autophagy (Additional file 1: Figure S4a,b). To further consolidate our conclusions, we examined the effect of MALAT1 on autophagy in the GC cell line BGC823. Treatment of CDDP $(10 \mu \mathrm{g} / \mathrm{ml})$ for $24 \mathrm{~h}$ induced a significant upregulation of MALAT1 (Additional file 1: Figure S4c) and activation of autophagy, while MALAT1 knockdown blunted the autophagic response to cisplatin (Additional file 1: Figure S4d) compared to cells transfected with a control vector. Ectopic expression of MALAT1 induced chemoresistance in SGC7901 cells as illustrated by decreased cleaved caspase-3 level in response to cisplatin (Additional file 1: Figure S4e). Knockdown of lncRNAATB did not have significant effect on the autophagy of SGC7901/VCR cells (Additional file 1: Figure S4f). These data suggest that knockdown of MALTA1 inhibits the autophagic response in GC cells.

To explore the mechanism by which MALAT1 was regulated, firstly, we found high enrichment of H3K27Ac at the promoter region of MALAT1 with UCSC Genome Bioinformatics (Additional file 1: Figure S5a). Furthermore, we found high enrichment of H3K27Ac in gastric cancer tissues compared with normal tissues at the promoter of MALAT1 (Additional file 1: Figure S5b). We speculate that histone acetylation activation might contribute to the upregulation of MALAT1 in gastric cancer tissues. Using the CHIP assay, the high enrichment of H3K27Ac was also found in the SGC7901/VCR cells compared to SGC7901 cells at the promoter of MALAT1 (Additional file 1: Figure S5c). To further consolidate our hypothesis, we treated SGC7901 cells with the histone deacetylase inhibitor trichostatin A (TSA). We found that MALAT1 was upregulated by the histone deacetylase inhibitor trichostatin A (TSA) (Additional file 1: Figure S5d). Taken together, histone acetylation is involved in the upregulation of MALAT1.

We found that the expression of MALAT1 were significantly upregulated in chemoresistant patients compared with that in chemosensitive patients (Additional file 1: Figure S6a). We did observe decreased expression of LC3B in chemosensitive patients compared with chemoresistant patients (Additional file 1: Figure S6a). According to data from the KMPlot database, we found that high MALAT1 expression resulted in a poorer disease-free survival (DFS, $n=153, p=0.049$ ) and overall survival (OS, $n=153, p=0,039)$ in patients who had received 5 -Fu-based adjuvant therapy (Additional file 1: Figure S6b).

\section{ATG12 is a downstream effector in MALAT1-mediated autophagy in GC cells}

Next, we sought to identify the underlying mechanism of MALAT1-mediated autophagy. First, using qRT-PCR, we determined the effect of MALAT1 on the expression of the identified regulators of autophagy ATG1, ATG2, 
ATG3, ATG4D, ATG4B, ATG4C, ATG5, ATG7, ATG12, ATG13, ATG14, BECN1, ULK1, LC3B, SQSTM1, STMN1, and RAB5A with qRT-PCR analysis. We demonstrated that MALAT1 silencing greatly reduced the mRNA level of ATG12 (Fig. 4a). Furthermore, western blot analysis illustrated that the suppression of MALAT1 substantially downregulated the protein level of ATG12; yet, its effect on ATG3 was not significant (Fig. 4b). Overexpression of MALAT1 increased both ATG12 mRNA and protein expression levels (Fig. 4c). As lncRNAs have been shown to be involved in multiple levels of genes regulation, including transcriptional regulation via recruitment of chromatinmodifying complexes, and post-transcriptional regulation by interactions with mRNAs, miRNAs, and proteins [7]. Previous studies have revealed that MALAT1 may exert biological effects via interacting with chromatinmodifying complexes, such as EZH2 [21, 22], by acting as competitive endogenous RNAs (ceRNAs) [22], or by affecting protein stability [10] or protein phosphorylation status [12]. As anticipated, RNA pull-down experiments revealed that MALAT1 specifically interacted with EZH2 (Fig. 4d). We then explored whether MALAT1 or EZH2 silencing had any effect on the transcript levels of polycomb repressive complex 2 (PRC2) targets. qRT-PCR analysis revealed that either MALAT1 or EZH2 silencing suppressed the mRNA levels of previously identified PRC2 targets [23], including CCDN2, BMP2, KLF4 and SERPINB2. However, knockdown of EZH2 had no significant effect on the transcript levels of ATG12, despite that a similar depression of these target genes being observed in EZH2-downregulated cells (Fig. 4e). Furthermore, knockdown of EZH2 resulted in decreased trimethylation level of H3K27 in the promoter regions of CCND2, BMP2 and KLF4 by ChIP-qPCR analysis. However, no significant change in the trimethylation level of H3K27 in the promoter region of ATG12 was observed (Fig. 4f). These data suggest that the effect of MALAT1 on ATG12 may not dependent on PRC2. Because MALAT1 regulates ATG12 transcriptionally, we hypothesized that MALAT1 may act as a miRNA sequestrant for regulating ATG12 expression.
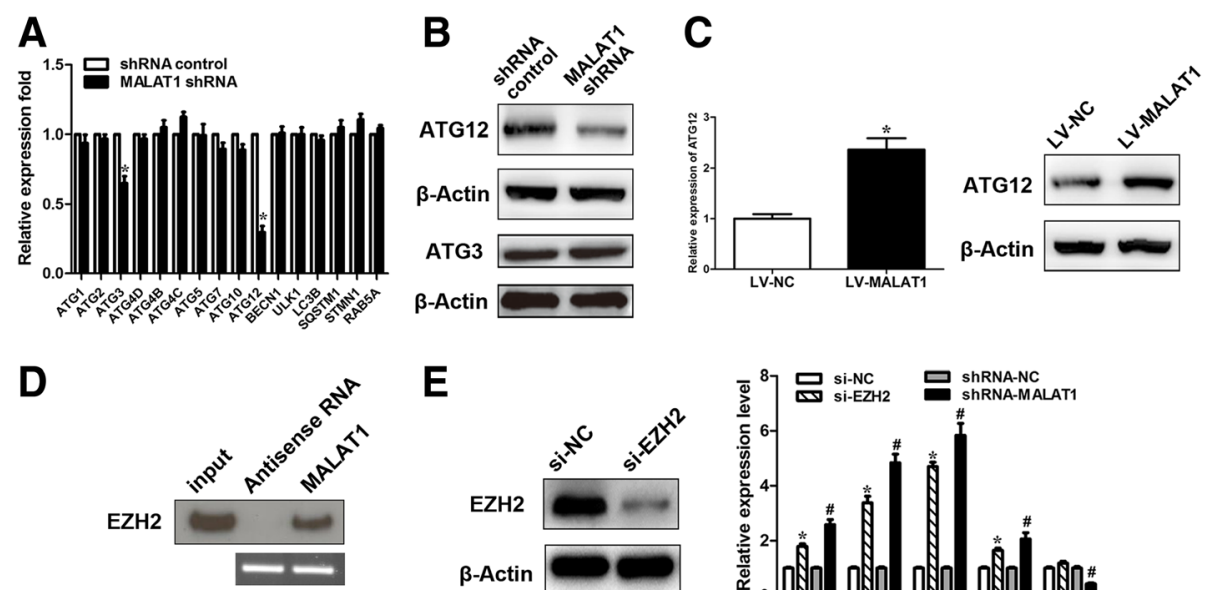

E
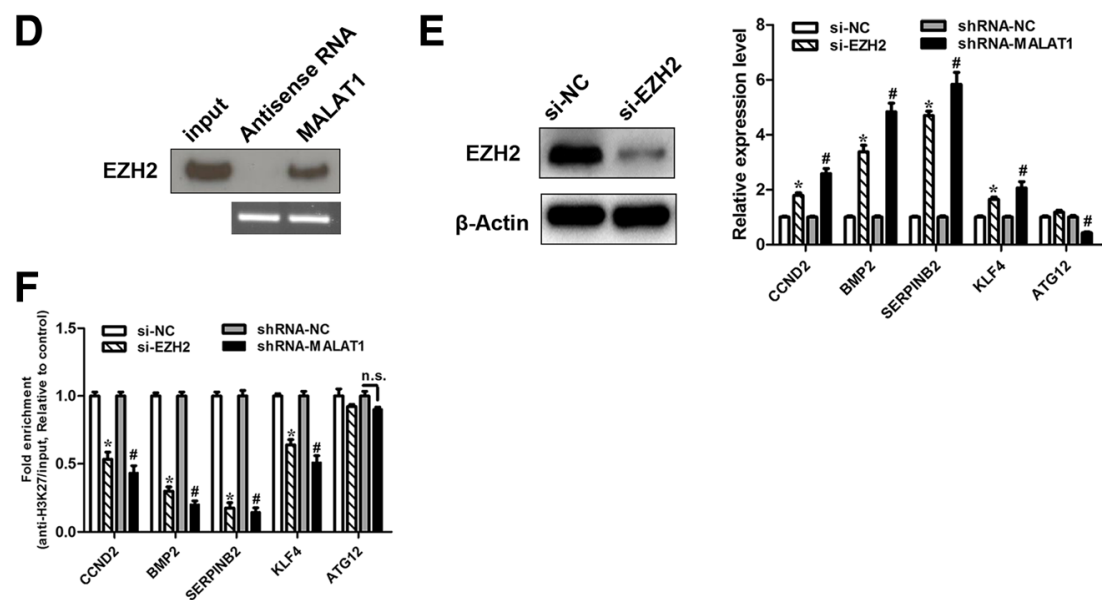

Fig. 4 MALAT1 regulates ATG12. a The mRNA expression of the indicated autophagy related genes was measured using real-time PCR in SGC7901NCR cells stably transfected with shRNA-MALAT1 or a control. Student $t$ tests were used to determine the statistical significance of the differences between the groups. $\mathbf{b}$ Western blot analysis of ATG12 and ATG3 was performed in SGC7901NCR cells stably transfected with shRNAMALAT1 or a control. c The mRNA or protein levels of ATG12 were determined using real-time PCR and Western blot analysis in SGC7901NCR cells stably transfected with shRNA-MALAT1 or a control. d Biotinylated MALAT1 or antisense RNA was incubated with cell extracts of SGC7901/ VCR cells, targeted with streptavidin beads, and washed, and the associated proteins were resolved on a gel. Western blot analysis detected the specific association of EZH2 and MALAT1 ( $n=3)$. e EZH2 knockdown efficiency was confirmed by Western blot. qRT-PCR analysis of putative PRC2 target genes after MALAT1 and EZH2 knockdown, respectively. f ChIP analysis of H3K27 trimethylation status of candidate EZH2 target genes after knockdown assay. The data are presented as the means \pm S.D. of values obtained in 3 independent experiments. \#, $p<0.05 .{ }^{*}, p<0.05$. n.S., not significant 
Bioinformatics analysis with starbase v2.0 revealed that MALAT1 formed complementary base pairing (two putative 7-mer complementary sequences) with miR-23b3p (Fig. 5a), which has been shown to target ATG12 and regulates autophagy-associated chemoresistance in gastric cancer in the previous study [24]. The expression level of MALAT1 was about one half of miR-23b-3p in SGC7901/ VCR cells (Additional file 1: Figure S7a). First, we examined whether MALAT1 was capable of interacting with miR-23b-3p. To confirm the physical interaction between MALAT1 and miR-23b-3p, we constructed luciferase reporter constructs (psicheck2). The luciferase reporter constructs were cotransfected with miR-23b-3p mimics or inhibitors into GC cells. As illustrated in Fig. 5b, miR-23b$3 \mathrm{p}$ mimics reduced the luciferase activity of the construct containing wild-type (WT) MALAT1. However, luciferase activity of constructs containing mutant MALAT1 was comparable to that of control cells. Our data indicate a direct interaction between MALAT1 and miR-23b-3p. We employed the biotin-avidin pull-down assay to determine whether miR-23b-3p could pull down MALAT1. SGC7901/VCR cells transfected with biotinylated miR-23b-3p
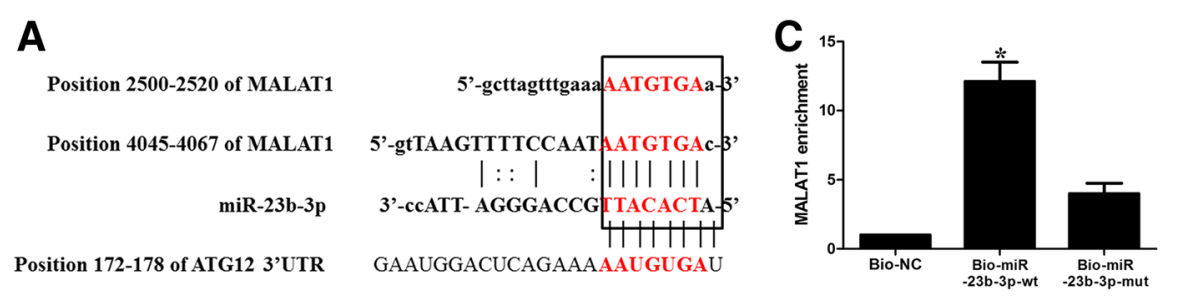

B
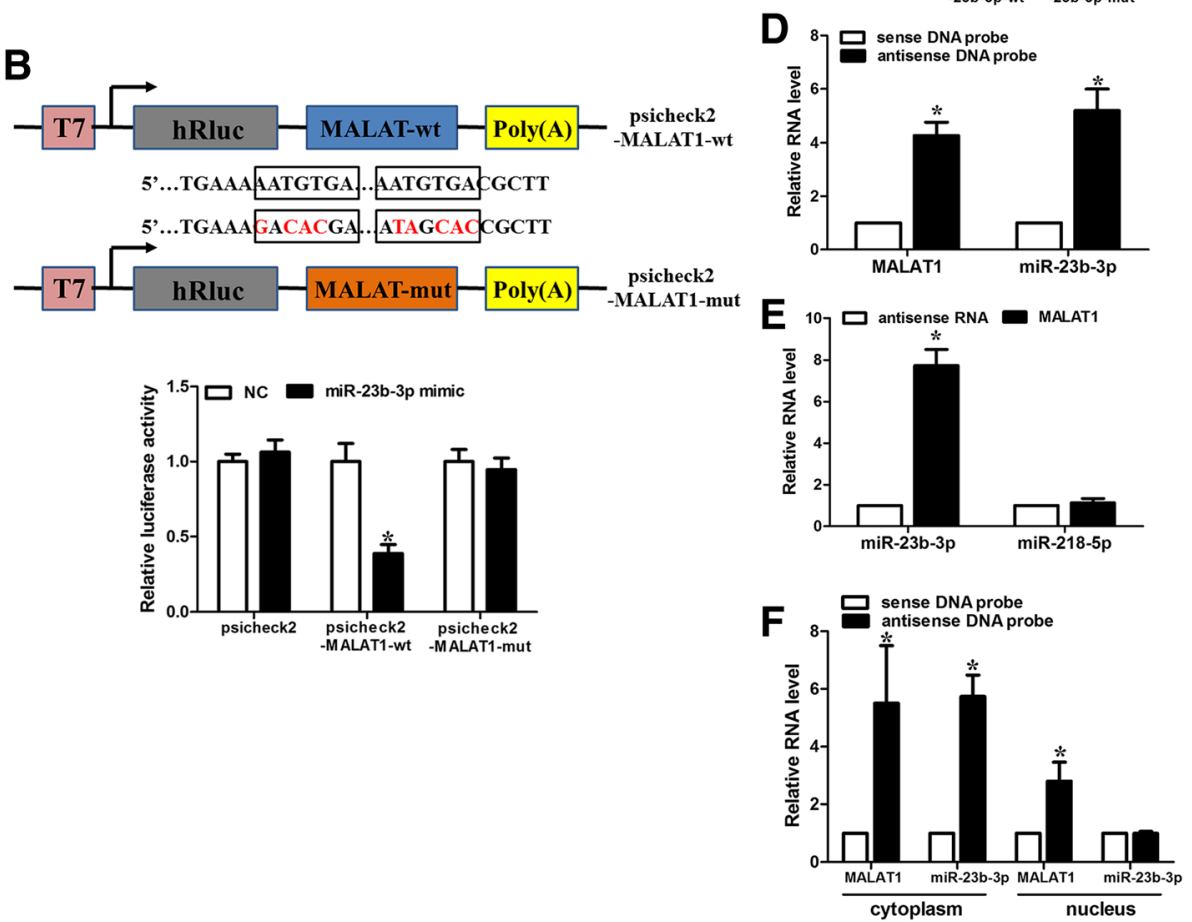

Fig. 5 MALAT1 is a molecular sponge for miR-23b-3p. a Illustration of the base pairing between miR-23b-3p and MALAT1. The base pairing between miR-23b-3p and ATG12 3'UTR is also shown. $\mathbf{b}$ Schematic representation of psicheck2-based luciferase reporter plasmid containing wild-type MALAT1 (psicheck2-MALAT1-wt) and a mutant reporter construct in which two putative miR-23b-3p binding sites were mutated (psicheck2-MALAT1-mut), and mutated bases are indicated in red. miR-23b-3p or control mimics were transfected into SGC7901/NCR cells together with the indicated psicheck2based luciferase reporter construct. Twenty-four hours after transfection, reporter activity was measured and plotted after normalizing with respect to Renilla luciferase activity. c miR-23b-3p can bind directly to MALAT1. SGC7901NCR cells were transfected with biotinylated wild-type miR-23b-3p (Bio-23b3p-wt) or biotinylated mutant miR-23b-3p (Bio-23b-3p-mut). A biotinylated miRNA that is not complementary to MALAT1 was used as a negative control (Bio-NC). Forty-eight hours after transfection, cells were harvested for biotin-based pull-down assay. MALAT1 expression levels were analysed by real-time PCR. *,$p<0.05$ versus Bio-NC. $\mathbf{d}$ Lysates from SGC7901NCR cells were incubated with in vitro-synthesized biotin-labeled sense or antisense DNA probes against MALAT1 for biotin pull-down assay, followed by real-time RT-PCR analysis to examine miR-23b-3p levels. e Lysates from SGC7901/NCR cells were incubated with in vitro-synthesized biotin-labeled MALAT1 and antisense RNA for biotin pull-down assay, followed by real-time RT-PCR analysis to examine miR-23b-3p and miR-218-5p levels. f SGC7901NCR cells were subjected to cytoplasm or nucleus fractionation before each fraction was incubated with in vitro-synthesized biotin-labeled sense or antisense DNA probes of MALAT1 for biotin pull-down assay, followed by real-time RT-PCR analysis to examine miR-23b-3p levels. Data shown are means \pm S.D. $\left(n=3 ;{ }^{*}, p<0.05\right.$, two-tailed $t$-test). ${ }^{*}, p<0.05$ 
were collected for the biotin-streptavidin pull-down assay. MALAT1 was pulled down and analyzed by qRT-PCR. As anticipated, miR-23b-3p successfully pulled down MALAT1; however, mutations in the binding site between $M A L A T 1$ and miR-23b-3p disturbed of the pull down of MALAT1 by miR-23b-3p (Fig. 5c). We also used in vitrosynthesized biotinylated $M A L A T 1$ probe and biotinylated antisense DNA probe-enriched endogenous MALAT1 to pull down miR-23b-3p. MiR-218-5p, which formed no base pairing with MALAT1, was used as a negative control. We showed that MALAT1 specifically pulled down miR-23b-3p (Fig. 5d and e), however, MALAT1 was not able to pull down miR-218-5p (Fig. 5e). These data confirmed that MALAT1 physically interacts with miR-23b$3 \mathrm{p}$ in a sequence-specific manner. Next, we chose to test where the interaction of MALAT1 with miR-23b-3p occurs. The results revealed that miR-23b-3p was mainly expressed in the cytoplasm, while MALAT1 was located in both the nuclear and cytoplasmic fractions of GC cells (Additional file 1: Figure S7b). The antisense DNA probe enriched endogenous MALAT1 pull down of miR-23b-3p from the cytosolic but not the nuclear fraction (Fig. 5f). Furthermore, treatment of CDDP $(10 \mu \mathrm{g} / \mathrm{ml})$ for $24 \mathrm{~h}$ induced a downregulation of miR-23b-3p in GC cells (Additional file 1: Figure S8a).

In the previous study, An et al [24] demonstrated that ATG12 is a bona fide target of miR-23b-3p and that its expression can be regulated by miR-23b-3p in GC. Finally, given that miR-23b-3p was capable of targeting both MALAT1 and ATG12, we tested whether MALAT1 could competitively sequester miR-23b-3p and relieves the inhibitory effect of miR-23b-3p on ATG12. qRTPCR analysis revealed that MALAT1 silencing increased, whereas ectopic expression of MALAT1 increased the levels of miR-23b-3p (Fig. 6a). miR-23b-3p had no effect on the expression level of MALAT1 (Additional file 1: Figure S8b). As MALAT1 shares regulatory miR-23b-3p with ATG12, we wanted to determine if MALAT1 regulates ATG12 through its regulatory role on miR-23b-3p. Western blot analysis showed that MALAT1 overexpression attenuated the decrease in the protein expression levels of ATG12 induced by miR-23b-3p mimics (Fig. 6b). In addition, the miR-23b-3p inhibitor-mediated upregulation of ATG12 was greatly reversed by MALAT1 knockdown (Fig. 6c). Furthermore, overexpression of miR-23b-3p binding-defective MALAT1 had no significant effect on the expression of ATG12 (Fig. 6d). To reinforce the conclusion, we constructed a luciferase reporter containing 3'-UTR of ATG12. The reporter activity was obviously suppressed by MALAT1 silencing, while the miR-23b-3p inhibitor relieved this decrease (Fig. 6e). In contrast, the overexpression of WT MALAT1, but not the miR-23b-3p binding-defective $M A L A T 1$, increased the reporter activity, while miR-23b-3p mimics attenuated this increase (Fig. 6f). Taken together, these results suggest that MALAT1 competitively sequesters miR-23b-3p and attenuates the inhibitory effect of miR-23b-3p on ATG12, thereby elevating the expression of ATG12. To explore the possibility that MALAT1 might function through modulating other miR-23b-3p target genes, we examined the effect of MALAT1 on HMGB2, which has been shown to regulate chemore sistance-associated autophagy [24]. We found that MALAT1 silencing suppressed the expression of ATG12 and HMGB2, whereas co-transfection of miRNA-23b-5p inhibitor attenuated this inhibition (Additional file 1: Figure S8c). The incomplete rescue of HMGB2 by miRNA-23b-3p inhibitor suggests that other mechanisms might also be involved in its regulation.

\section{MALAT1 promotes autophagy associated chemoresistance of GC cells via miR-23b-3p}

Next, we sought to identify whether MALAT1 associated chemoresistance was dependent on ATG12 upregulation. In the previous study, ATG12 silencing obviously increased chemosensitivity in GC cells and miR-23b-3p chemosensitizes GC cells by regulating ATG12 [24]. In the present study, we showed that ectopic expression of ATG12 alleviated the suppressive effect on autophagy induced by MALAT1 silencing (Fig. 7a). Additionally, we demonstrated that the chemosensitization induced by MALAT1 suppression could be ameliorated by ATG12 overexpression (Fig. 7b). We also found that miR-23b-3p inhibitors relieved the inhibition of autophagy caused by MALAT1 suppression (Fig. 7c). Similarly, miR-23b-3p inhibitors abrogated the chemosensitization induced by MALAT1 knockdown (Fig. 7b). Furthermore, the activation of autophagic response induced by MALAT1 overexpression could be ameliorated by miR-23b-3p inhibitors (Fig. 7d). These data suggest that MALAT1 promotes autophagy-associated chemoresistance of GC cells via sequestration of miR-23b-3p.

We employed the xenograft model to confirm the effects of MALAT1 and miR-23b-3p on chemosensitivity. Consistent with in vitro observations, we observed that while chemotherapeutics greatly decreased tumor volume, MALAT1 overexpression significantly increased the chemoresistance (Fig. 8a, b). The drug resistance induced by MALAT1 overexpression could be reversed by ectopic miR-23b-3p expression (Fig. 8a, b). Furthermore, western blot analysis revealed that the tumor LC3-II to LC3-I ration from the miR-23b-3p overexpression group was lower, which was rescued by overexpression of MALAT1 (Fig. 8c). A summary diagram presenting the interaction between MALAT1, miR-23b-3p, ATG12 and their effect on autophagy-associatated chemoresistance is shown in Fig. 8d. Furthermore, we found that the expression of miR-23b-3p was downregulated in chemoresistant (Additional file 1: Figure S9a) patients, whereas the 

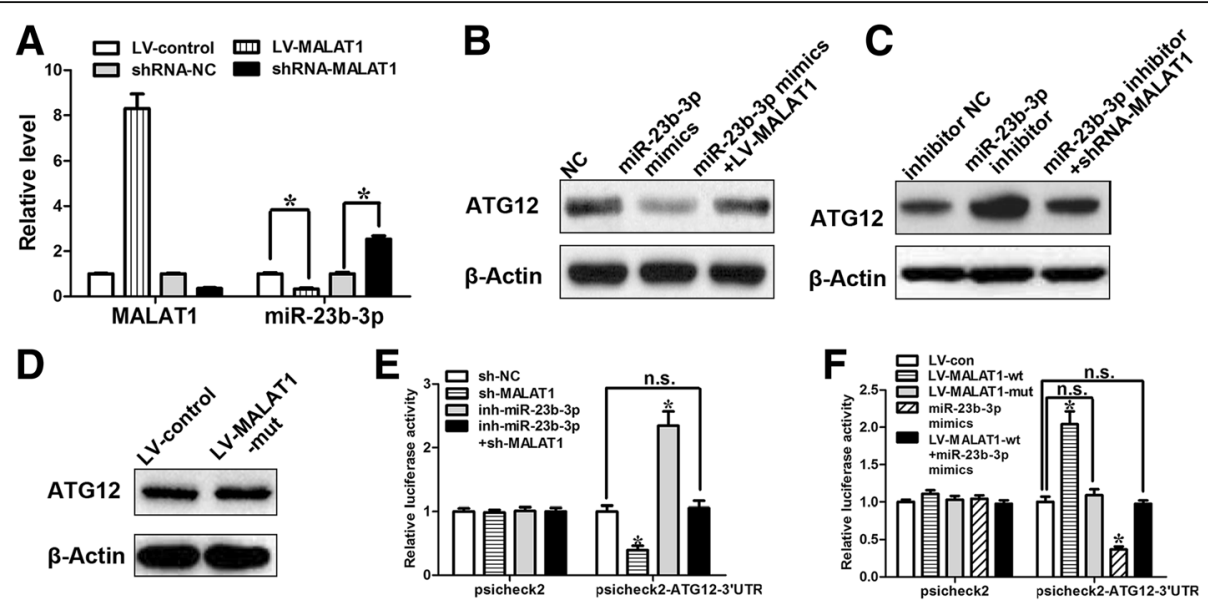

Fig. 6 MALAT1 relieves the inhibitory effect of miR-23b-3p on ATG12. a SGC7901/NCR cells were infected with lentiviruses expressing control shRNA or MALAT1 shRNA. Forty-eight hours after infection, total RNA was subjected to real-time RT-PCR analysis. $\mathbf{b}$ The protein levels of ATG12 in SGC7901NCR cells transfected with mimic control, miR-23b-3p mimics, miR-23b-3p mimics +LV-MALAT1. c The protein levels of ATG12 in SGC7901NCR cells transfected with inhibitor control, miR-23b-3p inhibitor, miR-23b-3p inhibitor+shRNA-MALAT1. d Overexpression of miR-23b-3p binding-defective MALAT1 had no significant effect on the expression of ATG12 in SGC7901NCR cells. e, $\mathbf{f}$ Luciferase activity in SGC7901/NCR cells transfected with luciferase reporters containing ATG12 3'-UTR or nothing. Data are represented as the relative ratio of firefly luciferase activity to Renilla luciferase activity. Error bars represent the mean \pm S.D. of triplicate experiments. ${ }^{*}, p<0.05$

expression of ATG12 was increased in chemoresistant patients. According to data from The KMPlot database (TCGA), patients with low miR-23b-3p expression (Additional file 1: Figure S9b) and high ATG12 expression (Additional file 1: Figure S9c) had a decreased DFS and OS in patients who received 5-Fubased adjuvant therapy.

\section{Discussion}

Chemotherapy remains the first line of therapy for advanced/metastatic GC. Chemoresistance, whether primary or acquired, is the main obstacle in the majority of cancers. ${ }^{5}$ Although great efforts have been taken into clarifying the molecular mechanisms of the chemoresistance [5], the precise mechanisms remain largely unknown. Autophagy, an

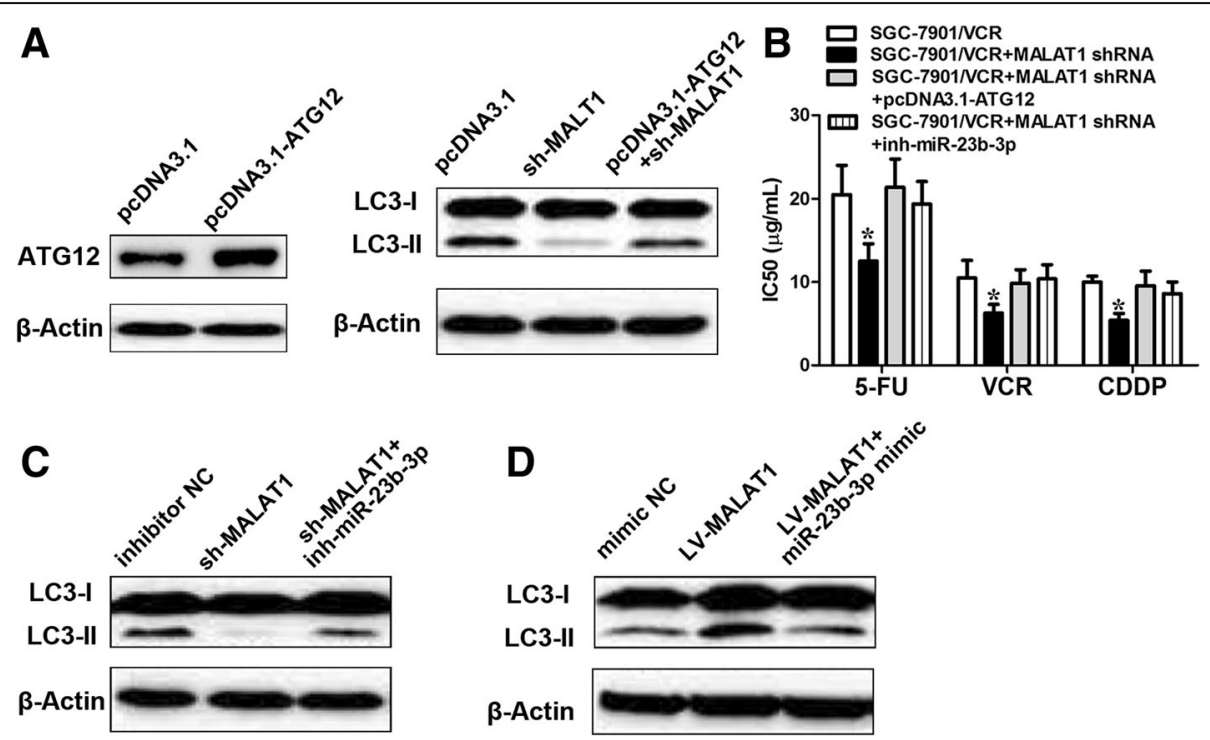

Fig. 7 MALAT1 regulates autophagy via ATG12. a Western blot analysis to confirm the efficacy of overexpression of ATG12. SGC7901/VCR cells were transfected with pCDNA3.1- empty vector, shRNA-MALAT1, shRNA-MALAT1+ pcDNA3.1-ATG12. LC3-II expression was evaluated by Western blot. b The decreased IC50 values induced by MALAT1 knockdown could be relieved by ATG12 or miR-23b-3p inhibitors. c SGC7901/NCR cells were transfected with inhibitor NC, shRNA-MALAT1, shRNA-MALAT1+ miR-23b-3p inhibitor. LC3-II expression was evaluated by Western blot. $\mathbf{d}$ SGC7901/NCR cells were transfected with inhibitor NC, LV-MALAT1, LV-MALAT1+ miR-23b-3p mimics. LC3-II expression was evaluated by Western blot. Data shown are means \pm S.D. $\left(n=3\right.$; two-tailed $t$-test). ${ }^{*}, p<0.05$ 


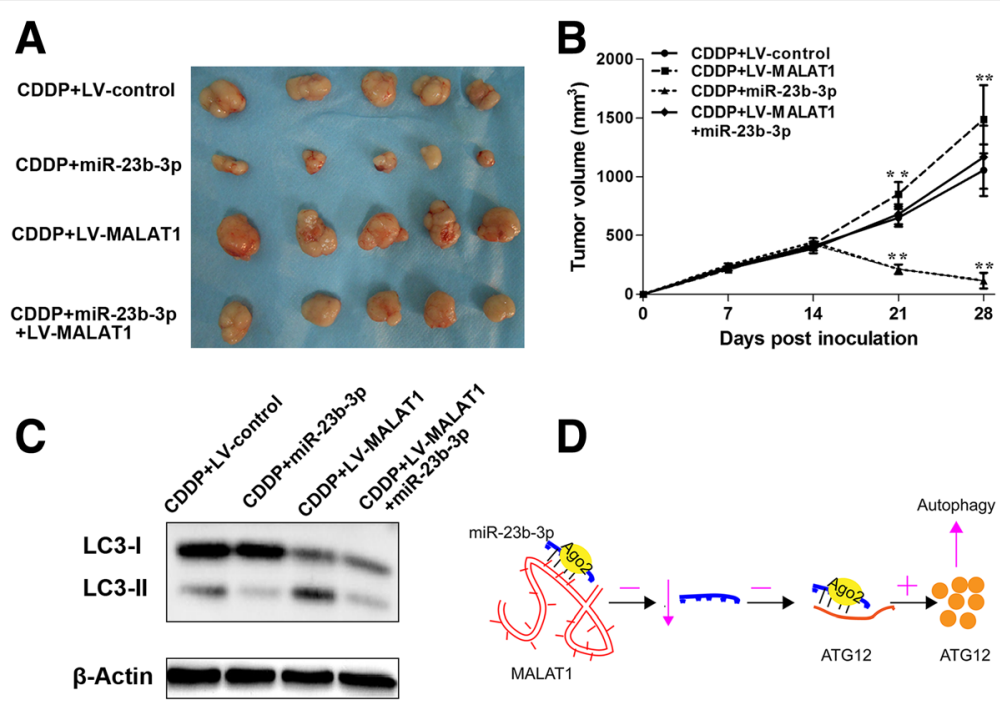

Fig. 8 The restoration of miR-23b-3p reversed the drug resistance induced by MALAT1 overexpression in vivo. $1.0 \times 10^{7}$ SGC7901/NCR cells stably transfected with lenti-MALAT1 or lenti-NC or lenti-MALAT1+miR-23b-3p were subcutaneously injected into the flank of nude mice. Two weeks later, the mice were intraperitoneally injected with PBS containing CDDP $(10 \mathrm{mg} / \mathrm{kg})$ once per week. The mice were humanely killed on day 28 , and the tumors were measured and photographed. Tumor volumes (a) and tumor growth curves (b) of subcutaneous implantation models of GC are shown. c Total protein fractions were isolated from cells derived from 3 representative xenograft samples from each group, and the LC3 proteins were detected by Western blot analysis. Data shown are means \pm S.D. $\left(n=3 ;{ }^{* *}, p<0.01\right.$, two-tailed $t$-test). $\mathbf{d}$ A summary diagram presenting the interaction between MALAT1, miR-23b-3p, ATG12 and their effect on autophagy-associatated chemoresistance

evolutionarily conserved, lysosome-mediated intracellular degradation system that is important for cellular homeostasis, development and differentiation [17], has emerged as a new player in chemoresistance [6]. Accumulating evidence has demonstrated that IncRNAs contribute to the chemoresistance in a wide ranges of cancers $[7,25,26]$. In the present study, we bridged the gap between lncRNA and autophagy associated chemoresistance in GC. We showed that MALAT1 competes with ATG12 mRNA for miR-23b$3 \mathrm{p}$ binding and ameliorates the suppressive effect of miR23b-3p on ATG12.

Previous studies have contradictory opinions about the roles of autophagy in cancer progression. Qu et al [27] showed that disruption of autophagy may promote the tumorigenesis. Autophagy has also been shown to protect cells from stress conditions, such as starvation, chemotherapeutics or radiotherapeutics [6, 17, 24]. Various kinds of stimuli, including anticancer cancer treatment, hypoxia and starvation may induce autophagy [16, 17, 24, 28]. Chemotherapy-induced autophagy have been revealed to help cancer cells escape from deadly cell damage, thereby contributing to chemoresistance [17, 24]. Thus, targeting autophagy-associated regulators may be a potential strategy for eliminating therapeutic resistance in cancer.

LncRNAs have been demonstrated as potent prognostic indicators in various cancers $[8,9,29]$. A growing volume of literature illustrates the association between treatment efficacy (chemotherapy or radiotherapy sensitivity) and tumor lncRNA expression [7, 25, 26]. A few studies that have also established a link between lncRNAs and autophagy-associated chemoresistance [30, 31].

Our study showed that MALAT1, a lncRNA overexpressed in a wide range of cancers [11,29,32], was upregulated in chemoresistant GC cells. Some studies have revealed that MALAT1 may be an inducer of autophagy [33-35], few studies have concentrated on the association between MALAT1 and chemosensitivity. Thus, its role in chemoresistance requires further research. In the present study, we explored the effect of MALAT1 on chemosensitivity in GC cells. We showed that MALAT1 competitively sequesters miR-23b-3p and relieves the inhibitory effect of miR-23b-3p on ATG12, thereby increasing the expression of ATG12. ATG12, a vital regulator of autophagy, is upregulated in various cancers $[17,24,36]$. Our data revealed that the chemosensitizing effect of MALAT1 knockdown was independent of proliferation. However, one study reported that MALAT1 promotes pancreatic cancer proliferation via the stimulation of autophagy [34]. Previous studies have also showed that CQ-mediated autophagy suppression inhibited cell proliferation in pancreatic cancer cell lines [37, 38]. However, this contradicts our data, as chemoresistant GC cells and the parental cells demonstrated similar proliferation rates in the absence of chemotherapeutics. Additionally, it is worth noting that similar reports were primarily on pancreatic cancer, which have higher levels of basal autophagy than other 
types of cancer [37], suggesting that autophagy-associated cell proliferation is cancer specific and requires intensive investigation.

Overall, our study identified the role of MALAT1 in the chemoresistance of GC. MALAT1 promotes autophagy by sequestering miR-23b-3p and tittering miR-23b-3p off its target ATG12, thus increasing the level of ATG12 and contributing to autophagy-associated chemoresistance. Our report provides novel insights into the molecular mechanisms underlying chemoresistance.

\section{Additional files}

Additional file 1: Figure S1. Flow cytometric analysis of Annexin $\mathrm{V}$ staining. Figure S2 MALAT1 expression levels vary across different cancer types in the TCGA database. Figure S3 (a) MALAT1 expression was detected in SGC7901 NCR cells by qRT-PCR after transduction of lentiviruses encoding MALAT1 shRNA or a scrambled shRNA. Northern blot analysis of MALAT1 expression in SGC7901NCR cells after transduction of lentiviruses encoding MALAT1 shRNA or a scrambled shRNA. (b) MALAT1 expression was detected in SGC7901NCR cells by qRT-PCR after transfection of lentivirus harboring the full-length human MALAT1 sequence or the empty vector. The data are presented as the means \pm S.D. of values obtained in 3 independent experiments. *, $p<0.05$. Figure S4 MALAT1 promotes autophagy. (a) SGC7901/NCR cells stably transfected with full-length human MALAT1 sequence or the empty vector were subjected to Western blot analysis of LC3-II and p62. (b) Autophagy was evaluated using transmission electron microscopy in SGC7901/NCR stably transfected with full-length human MALAT1 sequence or the empty vector. (c) In BGC823 cells, treatment of CDDP $(10 \mu \mathrm{g} / \mathrm{ml})$ for $24 \mathrm{~h}$ induced a significant upregulation of MALAT1 as determined with qRT-PCR analysis. (d) In BGC823 cells, treatment of CDDP $(10 \mathrm{\mu g} / \mathrm{ml})$ for $24 \mathrm{~h}$ induced a significant activation of autophagy, while MALAT1 knockdown blunted the autophagic response to cisplatin. (e) SGC7901 cells transfected with full-length human MALAT1 sequence or the empty vector were treated with the cisplatin $(5 \mu \mathrm{g} / \mathrm{ml})$ for $24 \mathrm{~h}$, caspase-3 protein was detected by Western blot. The data are presented as the means \pm S.D. of values obtained in 3 independent experiments. ${ }^{*}, p<$ 0.05. (f) SGC7901/NCR cells stably transfected with shRNA-IncRNA-ATB or a control were subjected to Western blot analysis of LC3-II and p62. Figure S5 (a) UCSC Genome Bioinformatics Site (http://genome.ucsc. edu/) showed high enrichment of H3K27Ac at the promoter of MALAT1. (b) ChIP assays detected the H3K27Ac acetylation at promoter of MALAT1 in gastric cancer tissues. (c) ChIP assays detected the H3K27Ac acetylation at promoter of MALAT1 in gastric cancer cells.*, $p<0.05,{ }^{* *}, p<0.01$. (d) The expression of the MALAT1 transcript (mean \pm standard deviation) was detected using RT-PCR after cells were stimulated with varying concentrations of the histone deacetylase inhibitor trichostatin A (TSA) for 24 hr. Figure S6 (a) Compared with chemosensitive patients, LC3B and MALAT1 were markedly upregulated in chemoresistant patients using immunohistochemical analysis (for LC3B) and in situ hybridization analysis (for MALAT1). (b) According to data from The KMPlot database (TCGA), high MALAT1 expression resulted in a poorer disease-free survival (DFS, $n=153, p=0.049$ ) and overall survival (OS, $n=153, p=0,039$ ) in patients who received 5 -Fu based adjuvant therapy. The HRs and $p$ values were calculated with log-rank tests. Figure S7 (a) The copy number of MALAT1 or miR-23b-3p detected SGC7901NCR cells, using RT-PCR and standard curves of known copy numbers of plasmid-derived reference standard. Error bars show standard deviation. (b) Cellular characterization of MALAT1 and miR23b-3p, the levels of nuclear control transcript (U1), cytoplasmic control transcript (Actin mRNA), and MALAT1 were assessed by qRT-PCR in nuclear and cytoplasmic fractions in SGC7901/NCR cells. Data are presented as a percentage of U1, Actin and MALAT1 levels and total levels for each were taken to be $100 \%$. The data are presented as the means \pm S.D. of values obtained in 3 independent experiments. Figure $\mathbf{S 8}$ (a) In BGC823 cells, treatment of CDDP $(10 \mu \mathrm{g} / \mathrm{ml})$ for $24 \mathrm{~h}$ induced a significant downregulation of miR-23b-3p as determined with qRT-PCR analysis. (b) MALAT1 expression was detected in SGC7901NCR cells by qRT-PCR after transduction of lentiviruses encoding miR-23b-3p mimic or control mimic. (c) SGC7901NCR cells were transfected with sh-NC, sh-MALAT1, sh-MALAT1 +miRNA-23b-3p inhibitor and miR-23b-3p inhibitor. qRT-PCR was performed $48 \mathrm{~h}$ post transfection. ATG12 and HMGB2 were determined with gRT-PCR analysis. Figure S9 (a) Compared with chemosensitive patients, ATG12 was markedly upregulated and miR-23b-3p was downregulated in chemoresistant patients using immunohistochemical analysis (for ATG12) and in situ hybridization analysis (for miR-23b-3p). (b,c) According to data from The KMPlot database (TCGA), low miR-23b-3p and high ATG12 expression resulted in a poorer disease-free survival and overall survival in patients who received 5-Fu based adjuvant therapy. The HRs and $p$ values were calculated with log-rank tests. Table S1 Primer sequence used in this study. (DOCX $2642 \mathrm{~kb}$ )

Additional file 2: Suppemental materials and methods. (DOCX $16 \mathrm{~kb}$ )

\section{Abbreviations}

GC: Gastric cancer; HOTAIR: HOX transcript antisense RNA; IncRNA: Long noncoding RNA; MALAT1: Metastasis-associated lung adenocarcinoma transcript 1

\section{Acknowledgments}

This work was supported by the National Nature Science Foundation of China (Grant Nos. 81702388); Zhejiang province key science and technology innovation team (Grant Nos. 2013TD13); Institute of Gastroenterology, Zhejiang University (IGZU); Wenzhou Science and Technology Bureau (Grant Nos. Y20160426).

\section{Authors' contributions}

HYR, YYC and CYF carried out the molecular genetic studies, participated in the sequence alignment and drafted the manuscript. YSW, LKQ and TXC carried out the immunoassays. CSR participated in the sequence alignment. HYRCED, LXZ and CYF participated in the design of the study and performed the statistical analysis. HYR and CYF conceived of the study and participated in its design and coordination. All authors read and approved the final manuscript.

\section{Competing interests}

The authors declare that they have no competing interests.

\section{Publisher's Note}

Springer Nature remains neutral with regard to jurisdictional claims in published maps and institutional affiliations.

\section{Author details}

${ }^{1}$ Department of General Surgery, The third Clinical Institute Affiliated to Wenzhou Medical University, Wenzhou People's Hospital, Wenzhou, Zhejiang, China. ${ }^{2}$ Department of Gastroenterology, The third Clinical Institute Affiliated to Wenzhou Medical University, Wenzhou People's Hospital, Wenzhou, Zhejiang, China. Institute of Gastroenterology, Zhejiang University(IGZJU), Hangzhou, Zhejiang, China. ${ }^{4}$ Department of radiology, Wenzhou No.3 Clinical Institute of Wenzhou Medical University, Wenzhou People's Hospital, No. 57 Canghou Street, Wenzhou, Zhejiang 325000, China.

Received: 19 June 2017 Accepted: 13 November 2017 Published online: 21 November 2017

\section{References}

1. Siegel RL, Miller KD, Jemal A. Cancer statistics, 2015. CA Cancer J Clin. 2015;65:5-29.

2. Chen W, Zheng R, Baade PD, Zhang S, Zeng H, Bray F, et al. Cancer statistics in China, 2015. CA Cancer J Clin. 2016;66:115-32.

3. Karimi P, Islami F, Anandasabapathy S, Freedman ND, Kamangar F. Gastric cancer: descriptive epidemiology, risk factors, screening, and prevention. Cancer Epidemiol Biomarkers Prev. 2014;23:700-13.

4. Dassen AE, Dikken JL, van de Velde CJ, Wouters MW, Bosscha K, Lemmens VE. Changes in treatment patterns and their influence on long-term survival in patients with stages I-III gastric cancer in The Netherlands. Int J Cancer. 2013;133:1859-66.

5. Rebucci M, Michiels C. Molecular aspects of cancer cell resistance to chemotherapy. Biochem Pharmacol. 2013;85:1219-26. 
6. Huang Z, Zhou L, Chen Z, Nice EC, Huang C. Stress management by autophagy: Implications for chemoresistance. Int J Cancer. 2016;139:23-32.

7. Qu L, Ding J, Chen C, Wu ZJ, Liu B, Gao Y, et al. Exosome-Transmitted IncARSR Promotes Sunitinib Resistance in Renal Cancer by Acting as a Competing Endogenous RNA. Cancer Cell. 2016;29:653-68.

8. Zheng J, Huang X, Tan W, Yu D, Du Z, Chang J, et al. Pancreatic cancer risk variant in LINC00673 creates a miR-1231 binding site and interferes with PTPN11 degradation. Nat Genet. 2016;48:747-57.

9. Ma MZ, Zhang Y, Weng M, Wang SH, Hu Y, Hou ZY, et al. Long non-coding RNA GCASPC, a target of miR-17-3p, negatively regulates pyruvate carboxylase-dependent cell proliferation in gallbladder cancer. Cancer Res. 2016;76:5361-71.

10. Luo F, Liu X, Ling M, Lu L, Shi L, Lu X, et al. The IncRNA MALAT1, acting through HIF-1a stabilization, enhances arsenite-induced glycolysis in human hepatic L-02 cells. Biochim Biophys Acta. 1862;2016:1685-95.

11. Latorre E, Carelli S, Raimondi I, D'Agostino V, Castiglioni I, Zucal C, et al. The Ribonucleic Complex HuR-MALAT1 Represses CD133 Expression and Suppresses Epithelial-Mesenchymal Transition in Breast Cancer. Cancer Res. 2016;76:2626-36.

12. Yao J, Wang $X Q$, Li YJ, Shan $K$, Yang $H$, Wang $Y N$, et al. Long non-coding RNA MALAT1 regulates retinal neurodegeneration through CREB signaling. EMBO Mol Med. 2016;8:346-62.

13. Michalik KM, You X, Manavski Y, Doddaballapur A, Zörnig M, Braun T, et al. Long noncoding RNA MALAT1 regulates endothelial cell function and vessel growth. Circ Res. 2014;114:1389-97.

14. Shang Y, Zhang Z, Liu Z, Feng B, Ren G, Li K, et al. miR-508-5p regulates multidrug resistance of gastric cancer by targeting $A B C B 1$ and ZNRD1. Oncogene. 2014;33:3267-76.

15. Xia L, Zhang D, Du R, Pan Y, Zhao L, Sun S, et al. miR-15b and miR-16 modulate multidrug resistance by targeting BCL2 in human gastric cancer cells. Int J Cancer. 2008;123:372-9.

16. Chen Y, Henson ES, Xiao W, Huang D, McMillan-Ward EM, Israels SJ, et al. Tyrosine kinase receptor EGFR regulates the switch in cancer cells between cell survival and cell death induced by autophagy in hypoxia. Autophagy. 2016;12:1029-46

17. Wang $P$, Zhang J, Zhang L, Zhu Z, Fan J, Chen L, et al. MicroRNA 23b regulates autophagy associated with radioresistance of pancreatic cancer cells. Gastroenterology. 2013;145:1133-43.

18. Johansen T, Lamark T. Selective autophagy mediated by autophagic adapter proteins. Autophagy. 2011:7:279-96.

19. Xiao H, Tang K, Liu P, Chen K, Hu J, Zeng J, et al. LncRNA MALAT1 functions as a competing endogenous RNA to regulate ZEB2 expression by sponging miR-200s in clear cell kidney carcinoma. Oncotarget. 2015;6:38005-15.

20. Ma J, Wang P, Yao Y, Liu Y, Li Z, Liu X, et al. Knockdown of long non-coding RNA MALAT1 increases the blood-tumor barrier permeability by upregulating miR-140. Biochim Biophys Acta. 1859;2016:324-38.

21. Fan $Y$, Shen B, Tan M, Mu X, Qin Y, Zhang F, et al. TGF- $\beta$-induced upregulation of MALAT1 promotes bladder cancer metastasis by associating with suz12. Clin Cancer Res. 2014:20:1531-41.

22. Hirata H, Hinoda Y, Shahryari V, Deng G, Nakajima K, Tabatabai ZL, et al. Long Noncoding RNA MALAT1 Promotes Aggressive Renal Cell Carcinoma through Ezh2 and Interacts with miR-205. Cancer Res. 2015;75:1322-31.

23. Bracken AP, Dietrich N, Pasini D, Hansen KH, Helin K. Genome-wide mapping of Polycomb target genes unravels their roles in cell fate transitions. Genes Dev. 2006;20:1123-36.

24. An $Y$, Zhang $Z$, Shang $Y$, Jiang $X$, Dong J, Yu $P$, et al. miR-23b-3p regulates the chemoresistance of gastric cancer cells by targeting ATG12 and HMGB2. Cell Death Dis. 2015;56:e1766.

25. Fan $Y$, Shen B, Tan M, Mu X, Qin Y, Zhang F, et al. Long non-coding RNA UCA1 increases chemoresistance of bladder cancer cells by regulating Wnt signaling. FEBS J. 2014;281:1750-8.

26. Özeş AR, Miller DF, Özeş ON, Fang F, Liu Y, Matei D, et al. NF-kB-HOTAIR axis links DNA damage response, chemoresistance and cellular senescence in ovarian cancer. Oncogene. 2016;35:5350-61.

27. Qu X, Yu J, Bhagat G, Furuya N, Hibshoosh H, Troxel A, Rosen J, et al. Promotion of tumorigenesis by heterozygous disruption of the beclin 1 autophagy gene. J Clin Invest. 2003;112:1809-20.

28. Song YJ, Zhang SS, Guo XL, Sun K, Han ZP, Li R, et al. Autophagy contributes to the survival of CD133+ liver cancer stem cells in the hypoxic and nutrientdeprived tumor microenvironment. Cancer Lett. 2013:339:70-81.
29. Wang SH, Zhang WJ, XC W, Weng MZ, Zhang MD, Cai Q, et al. The IncRNA MALAT1 functions as a competing endogenous RNA to regulate MCL-1 expression by sponging miR-363-3p in gallbladder cancer. J Cell Mol Med. 2016:20:2299-308.

30. Xiong H, Ni Z, He J, Jiang S, Li X, He J, et al. LncRNA HULC triggers autophagy via stabilizing Sirt1 and attenuates the chemosensitivity of HCC cells. Oncogene. 2017 Feb 6; 10.1038/onc.2016.521. [Epub ahead of print]

31. Chen YM, Liu Y, Wei HY, Lv KZ, Large FPF. intergenic non-coding RNA-ROR reverses gemcitabine-induced autophagy and apoptosis in breast cancer cells. Oncotarget. 2016;7(37):59604-17.

32. Vassallo I, Zinn P, Lai M, Rajakannu P, Hamou MF, Hegi ME. WIF1 re-expression in glioblastoma inhibits migration through attenuation of non-canonical WNT signaling by downregulating the IncRNA MALAT1. Oncogene. 2016;35:12-21.

33. Li Z, Li J, Tang N. Long noncoding RNA MALAT1 is a potent autophagy inducer protecting brain microvascular endothelial cells against oxygenglucose deprivation/reoxygenation-induced injury by sponging miR-26b and upregulating ULK2 expression. Neuroscience. 2017;354:1-10.

34. Li L, Chen H, Gao Y, Wang YW, Zhang GQ, Pan SH, et al. Long Noncoding RNA MALAT1 Promotes Aggressive Pancreatic Cancer Proliferation and Metastasis via the Stimulation of Autophagy. Mol Cancer Ther. 2016;15(9):2232-43.

35. Yuan P, Cao W, Zang Q, Li G, Guo X, Fan J. The HIF-2a-MALAT1-miR-216b axis regulates multi-drug resistance of hepatocellular carcinoma cells via modulating autophagy. Biochem Biophys Res Commun. 2016;478(3):1067-73.

36. Cufí S, Vazquez-Martin A, Oliveras-Ferraros C, Corominas-Faja B, Urruticoechea A, Martin-Castillo B, et al. Autophagy-related gene 12 (ATG12) is a novel determinant of primary resistance to HER2-targeted therapies: utility of transcriptome analysis of the autophagy interactome to guide breast cancer treatment. Oncotarget. 2012;3:1600-14.

37. Yang S, Wang X, Contino G, Liesa M, Sahin E, Ying H, et al. Pancreatic cancers require autophagy for tumor growth. Genes Dev. 2011;25:717-29.

38. Zeilhofer HU, Mollenhauer J, Brune K. Selective growth inhibition of ductal pancreatic adenocarcinoma cells by the lysosomotropic agent chloroquine. Cancer Lett. 1989:44:61-6.

\section{Submit your next manuscript to BioMed Central and we will help you at every step:}

- We accept pre-submission inquiries

- Our selector tool helps you to find the most relevant journal

- We provide round the clock customer support

- Convenient online submission

- Thorough peer review

- Inclusion in PubMed and all major indexing services

- Maximum visibility for your research

Submit your manuscript at www.biomedcentral.com/submit
) Biomed Central 